\title{
Quimioterapia en los tumores malignos de la infancia
}

En el momento actual disponemos de cuatro métodos de tratamiento: cirugia, irradiaciones (ralioterapia), quimioterapia e inmunoterapia, que pueden usarse en forma aislada o en combinación, y cuyas indicaciones dependen fundamentalmente de la edad del paciente, sitio de origen, extensión inicial del tumor, lel tipo histológico y grado de inmadurez. Con los dos primeros métodos se obticne alrededor se un $40 \%$ de curaciones y con el agregado de la quimioterapia este porcentaje aumenta at $80 \%$ en algunos tumores (nefroblastoma (8), Sarcoma de Ewing (6), etc.).

Las indicaciones de los tres métoklos de tratamiento (Cirugía-Radioterapia y Quimioterapia) se han resumido en la Tabla No 1.

Tabla No 1

METODOS DE TRATAMIENTO

EN' CANCERES RFANTILES

\begin{tabular}{cccc}
$\begin{array}{c}\text { Grado Clinico } \\
\text { Patoldgico }\end{array}$ & Cirugla & Radioterapia & $\begin{array}{c}\text { Quimio- } \\
\text { teratia }\end{array}$ \\
\hline I & + & + & + \\
\hline II & 0 & - & + \\
\hline III & + & + & 0 \\
\hline IV & + & + & 0 \\
\hline
\end{tabular}

Indicación principal.

+ Inclicación complementaria.

- Centro de Oncología, Hospital Luis Calvo Mackenna.

Rev. Chilena de Pediatría, Vol. 47, No 1, 1976
Dr. Huuberto del Pozo* y Dra. Victoria Beresi*

La cirugía puede eliminar completamente cumores localicados (Grado $\mathrm{r}$ ) y curar enfermos en un 50 a $100 \%$, según el tipo de tamor. Ej.: Rabdomio-sarcoma (1) cura en un 50 a (60)\% $\mathrm{T}$. de Wilms y Neuroblastoma, sobre el $80 \%$.

La mayoria de estos tumores Grado I despućs de su extirpación completa se tratan además con Radioterapia y Quimioterapia para climinar complctamente rosiduos tumorales microscópicos que pueden quedar en el sitio del tumor de origren (Nefroblastoma) o en sitios distantes $\mathbf{E j}$.: Osteosarcoma, en que sc usa quimioterapia múltiple "profiláctica" de metástasis pulmonares.

Cuando el tumor localizado es de gran tamaño y no parece posible extirparlo completamente se usa la quimioterapia $y / 0$ Radioterapia previa con el objeto de disminuir su tamano, enseguida efectuar su extirpación comple. ta (Nefroblastona, Neuroblastoma, Rabdomiosarcoma, etc.).

En Jos numores malignos Grado il el tumor se extiende a los tejidos vecinos, con o sin compromiso de ganglios rogionales y que puecle o no ser extirpado complctamente. El tratamiento comprende generalmente radio $y$ quimioterapia múltiple, $\mathrm{Ej} .:$ T. de Wilms, Neuroblastoma R.M.S. etc., después de la cirugfar.

En los Grados inj (Tumor regional con compromiso extenso de los tejidos vecinos e invasión de los ganglios regionales); el tratariento más importante es la quimioterapia múltiple y prolongada. La cirugia y Radioterapia pue- 
den usarse, en forma secundaria, para el tratamiento de residuos tumorales.

Fn los Grados iv (Tumor con metástasis y en las leucentias), el tratamiento fundamen. tal es la quimioceratpia múltiple, excepto en liufoma de Burkitt en que sólo se usa Ciclofosfamida (9). Ini radioterapia puede usarse cono profiláctica o curativa de localizaciones en S.N.C. y además junto con la cirugia para tratar algunos tumores que quedan después de quimiotrapia. La quimioterapia puede ser carativa en porcentajes que varim según el tipo his olkinico de tumor: 2,4\% en Neuroblastoma (i), $72 \%$ en Enf. de Hodgkin (2) y en las lencemias linfoblásticas se olytiene una sobrevida de 50 ; 5 anos y con posible curacion de estus pirientes, pero en la mayoría de ellos con tumores generalisialos (IV) la qujmioterapia es incapaz de curarlos pero de todas mancras proxluce ana mejoria objetiva $y$ subjetiva importante, con desaparición de doIores, aumento del apetito y por consiguiente, del peso, etc, que se puckle prolongar durante varios meses $y$ les permite a veces realizar una vida normal turante este Iapso. Es la quimioterapia paliativa.

\section{Tabla No 2}

\section{FACTORES OUIE INFLUYFN EN LA EFECTIVIDAD DE LA QUIMIOTERAPIA}

1. Bstato Fisiotofico det huésped.

Edad del paçicnte

Estado nutritivo

Funcionamiento del higado y riñones

Jnmunidad

2. Caracteristicas bioldgicas del tumot

Localización y cxtensión inicial

Grado de diferenciación

Potencial de multiplicación celular

Vascularización del tunor

\section{Famacologia y Farmacodinamfa de drogas}

Especificielad

V́a, dosis y esqucma de administración

Absorción y distribucjón

Toxicidacl

Resistencia

Tratamientos previos

Arecanismo de acción (Clasificación de drogas)

Combinacion de drogas
El papel potencial ale la inmunoterapia (5) aun no ha sido explorado totaimente. Sin entbargo experimentos en animales y resultito; prcliminares en pacientes con letucenia (4) parecen indicar que los mecanismos inmunolígicos sólo puélen clestruir pequeñas cantidades de células malignas, máximo 10'. Su lunción principal seria la eliminación ale célu. las tumorates que quedin después de st rolticción inicial por uno o varios de los otros tres métodos de tralamiento (B) .

Estas dependen fundamentalmente del estato fisiológrico del huésped, de lis características biolégicas del tumor y de la firmacología y famacodinimia de las drogas ( 7$)$.

En d estacio fisiológico del huitsped debtmos considerar:

a) Lét edr.xl. Los enfermos menores de un ano toleran mal las drogas y presentan ton mayor frecuencia síntomas tóxicos, por lo que hay que disminuir la dosis, y no usarlas en greneral en el menor de 6 meses.

b) Estado nutitivo. En general hemos visto que los niños desnutridos presentan sinto. mas tóxicos frecuentes y de mayor intensidad gue los que tienen buen estado nutritiou.

c) El estado funcional del lígado y riñones, organos que metabolizan y eliminan estos medicamentos y si estan alteraclos puede el enfermo intoxicarse con una dosis normal de estos. Respecto al tumor es fundinental conocer:

a) Localización y extensión iniciat, de esto depende al nictodo de tratamiento que usiremos.

b) El grado de diferenciación celular, porque mientras más inmaduro es, mejor responde a la quimioterapia.

c) Su vascularización, ya que las drogats penetran mal en él si tiene poca irrigación sanguínen.

El tercer factor que influye en la efectividad del tratamiento son las drogas que se usarán. Se sabe que actualmente no existen drogas especificas para los tumores malignos, aunque algunas son más efectivas sobre ajgunos de ellos. Ej. Ciclofosfamida en T. de Burkitt, Actinomicina D sobre T. de Wilms. En general la selección de ellas depende de su sensi- 
bilidad preestablecida por estudios en animaless o en el hombre.

Su cfecto depende de:

a) Dosis. La terapéutica debe encontrar un camino difícil entre dosis bajas que son ineficases $y$ dosis demasiado fucrtes que son peligrosás, La gravedad de las enfermedades trataclas justifica los riesgos tomados. En generitl sc isan dosis máximas que tolere el paciente y discontinuas. $\mathrm{Ej}$.: T. de Burkitt, etc.

b) Vía de administración. Existen drogas cuyo efecto es mayor al administrarlas por vía endovenosa que oral: Metotrexato.

c) Esquema terapéutico. Se ha demostrado que los trat:umientos intermitentes y prolongados (6 meses a 3 años) tienen mayor efecto antitumoral y menor toxicidad e inmunosupresión en el paciente que los tratamientos continuos.

d) Combinación de clrogas. Los mejores resultados actuales del tratamiento de tumores malignos se deben al uso dé quimioterapia múltiple, cuyos fundamentos se verán más itclelante.

e) De la existencia de "santuarios farmacológicos" que son regiones del organismo (S.N.C.) o de grandes tumores, en que las ctrogas penetran en canticlades insuficientes. Para obviar este inconveniente se usan por vía intratecal (Metotrexato-Citosina arabinosido, Hidrocortisona) o medicamentos que atraviesan la barrera hematoencefálica (BC - NU - CCNU).

f) Resistencia. Las células tumorales arquieren resistencia a las clrogas por mutación. igual que los bacterios a los antibióticos, y esto se produce especialmente, si el enfermo se ha tratado en forma incompleta (dosis o tiempo insuliciente, una sola droga, etc.) .

Por último la quimioterapia produce efectos tóxicos también sobre los tejidos normales que se multiplican activamente: Tejilo hematopoyético, mucosa gastro intestinal, folículo piloso y gónadas, e inmunosupresión con los consiguientes peligros de aplasia medular e infecciones más frecuentes y graves en los enfermos. Mediclas rigurosas y vigorosas (transfusiones, antibióticos, itislamiento), limitan estas consecuencias.
Por estos problemas inherentes al uso de quimioterapia se justifica la existencia de un centro oncológico que cuente con los recursos necesarios para realizar en forma adecuada y oportuna esta terapia y trate sus complica. ciones eficazmente (6).

\section{CLASIFICACION DE LAS DROGAS}

Sc pueden clasificar según su acción sobre el ciclo celular y sobre el metabolismo de las céInlas tumorales.

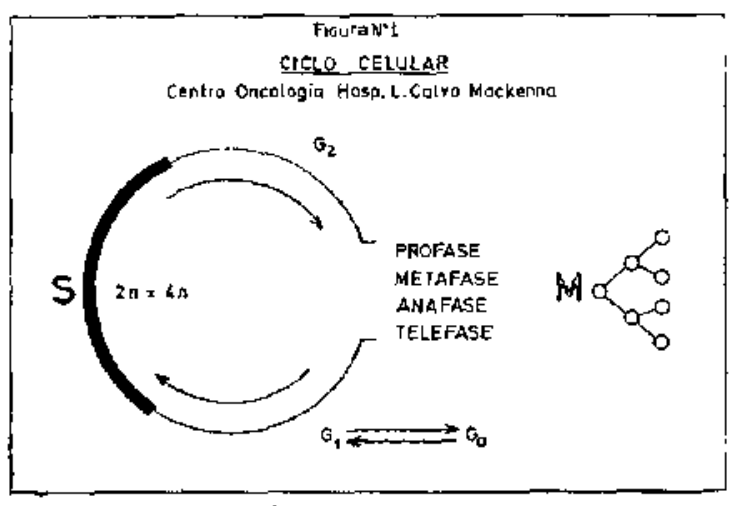

Figura, No l

El ciclo celular (figura 1) es lo que sucede entre una mitosis y la siguiente.

Li división celular ocurre en la mitosis (M) . F. tiempo de duplicación del material genético (2n a 4n) se denomina perfodo de sintesis (S). Los períodos entre ellas se conocen como periodos de presíntesis $\left(G_{1}\right)$ y postsintesis $\left(G_{n}\right)$.

La duración del tiempo que transcurre entre una mitosis y la siguiente se llama tiempo de generación (TG). La duración del tiempo que rcquiere una célula para pasar a través del ciclo celular se ha medido por Timidina tritiacla y vatra entre horas $(5$ a 10$)$ y dias, como consecuencia, la población celular se multiplica, en lo que se llama, forma asincrónica, es decir, en un mismo momento las células pueden encontrarse en todas las fases de este ciclo celular. Población celular sincronizada significa que todas las células están en una fase al mismo tiempo, mitosis por ejemplo. Existen ciertas drogas (Vincrostina, VM 126) que pueden producir la sincronización de las 
células en fase M y aumentar la proporción de celulas tumorales en fase $S, 18$ horas después de administrada, aumentando así la eficacia de una droga fase "dependiente" dada después de la Vincrostina.

Estudios experimentados realizados sobre cúlulas progenitoras de médula ósea normal, primariamente en estado $\mathrm{Go}$, y células cn multiplicación de una leucemia transplantada, tratadas con dosis crecicntes de una droga permiten observar tres curvas de muerte cclular. En la primera (figura 2), se observa una caída exponencial de las células sobrevivientes soguido por un plateau y aunque se aumente la dosis de la elroga no aumenta la antidad tle células destruidas.

E1 efecto 1ctal en las células de medula ósea es menor porque son pocas las que están en multiplicación. Se ha demostrado que cstas drogas actúan sólo en una fase del ciclo celular ( $\mathrm{S}$ o $M$ ) y se denominan por esto ageistes de fase especifica o fase dependiente. $\mathrm{Ej}$.: Vincristina-Vinblastina, antimetabolitos, antibióticos.
Estas drogas deben administrarse en forma repetida $y$ en intervalos definidos hasta que todas las células maligaas entren en la fase sensiłble y se expongan así a una concentración letal de ellas.

Una segunda curva se obtiene al usar otros agentes quimioterápicos en dosis crecientes.

Hay una destrucción exponencial de las células tumorales y normales, pero éstas, por su menor grado de multiplicación, se destruyen en menor cantidad. Estas drogas actúan sobre todo el ciclo celular y se denominan ciclo específicas. Ej.: Cicloforfamida, 5 Fluorucacilo, y es mejor administrartas en dosis máximas y con intervalos largos, para que dluésped so recupere de cualcuier efecto tóxico sobre sus tejiclos normales.

La tercera curva demuesira que la clestrucción de las rélulas tumorales y normales es igual, por consiguiente la acción letal de estos agentes es independiente del grado de multiplicación celu]ar. Son las drogas no específicas: mostaza nitrogenala. En la misna forma as-

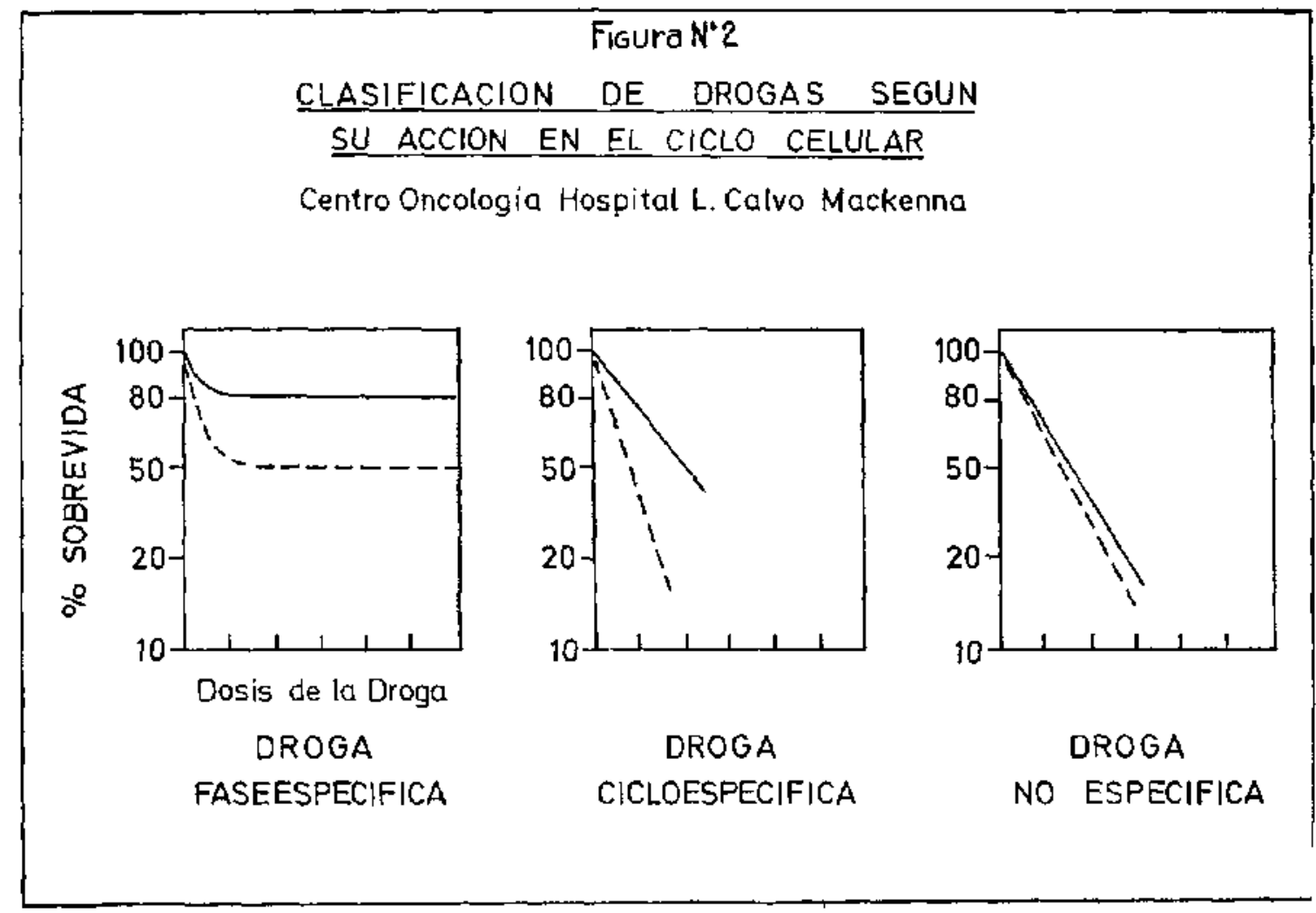


túan las radiaciones ionizantes. El uso de estos agentes está limitado a tumores localizados ya que si se usaran en tumores diseminados la dosis o la extensión de las irradiaciones sería letal para ambos: células tumorales y hućsped.

\section{AGENTES QUIMIOTERAPICOS (Tabla № 3)}

Sólo daremos una lista de los medicamentos más usados en cancerología infantil, con una breve descripción de su mecanismo de acción, sus indicaciones principales y su toxicidad más importante.

\section{ALQUILANTES}

La reacción química de la alquilación es el reemplazo de un átomo de $\boldsymbol{H}$ de una molécula por un grupo alquílico $\left(\mathrm{CH}_{2}\right)$. Su efecto le. tal parece deberse a la alquilación de cadenas nucleótidas, particularmente A.D.N. La mayorfa de estas trogas son ciclo específicas.

Las más usadas en Pediatria son:

Ciclofosfamida

BCNU-CCNU (Nitrosoureas)

Mostaza Nitrogenada

Clorambucil

\section{DROGAS}

INDICACJONES
TOXICIDAD ESPECIFICA

Leucoenccfalopatía
1. Antimetabolitos $\left(G_{t^{-}} S\right)$

- Metotrexato

- 6 Mercaptopurina

- Citarabina

- 5 Fluorutacilo

\section{Alquilantes}

- Ciclofosfamida (ciclnespecifico)

- BCNU.CCNU

\section{Anibioticos $\left(G_{x}-S-G_{2}\right)$}

$\longrightarrow$ Actinomicina D

- Adriamicina

4. Alcaloides (M)

-. Vincristina

- Vinblastina

5. Enximas

- L-Asparraginosa $\left(\mathrm{G}_{2}\right)$

Leucemia

Leuc.-Linf.-Tutnores embrionarios Lin formas - Histiacitosis

Leuccuia Osteosarcoma

eucemia

cucemia

I.cucemia-linfoma

Cistitis

Tumores embrionarios

Meduloblastoma

Wilns-Tumones embrionarios

Leuc. Linf. Tum. embrionarios

Potencia Rx

Cardiaca

Neurologiea

Id.

6. Corticoesteroides

- Prednisona
Cushing 
Ciclofosfamida: Esta es bioquímicamente inerte y sólo Ios productos que resultan de su me. tabolización en el higatlo son letales. Estos metabolitos, que se desconocen, se eliminan por los riñones y pucden alcanzar altas concentraciones en la vejiga. Usada sola (T. de Burkitc) o en combinación con otros medicamentos, tiene múltiples indicaciones: leucemias agudas, otros linfomas, Neuroblastoma, etc.

Sus efectos tóxicos son: Mielosupresión, habitualmente de corta duración y con poco compromiso de las plaquetas. Alopecia, nátuseas, vómitos y cistitis hemorrágica. Para prevenir esta complicación la ciclolosfamida rlebe darse en la mañana y el enfermo debe tomar 3.000 $\mathrm{ml} / \mathrm{m}^{2}$ o más de líquido en 2.l hrs. Si se produce henaturia debe suspenderse esta droga hasta que se normalice la orina. Si la hematuria recurre o persiste debe practicarse una cis. tografía para ver si hay fibrosis vesical y si ésta existe debe suspendersc definitivamente cste medicamento.

En adultos se ha descrito amenorrea y aspermia, lo que poxlría scr un problema para los niños que sobrevivan.

Las nitrosoureas (BCNU-CCYU) tiencu el interés que atraviesan la barrera hemato encefálica y por esto se usan en tratamiento de Meduloblastorna y Lipendimomas.

\section{ANTIMETABOLITOS ( $C_{y}$ y $\quad$ S.)}

Son sustancias de conititución muy parccida a las de los metabolitos a coenzimas indispensables de la sintesis bioquínica e impiden ésta por un mecanismo de competición.

La acción de estos análogos es, en general, lenta por lo que su administración debe ser prolongada.

Los principales son:

Ametopterina (Mctotrexato). Se usa desde 1947 y es un antifólico, jnhibe la reductasa dihydrofolato e impide la conversión del ácido fólico en tetrahydrofolato y así interfiere en la sintesis de las pirimidinas.

El Metotrexato, administrado en dosis convencionales 20 a $40 \mathrm{mg} / \mathrm{m}^{2}$, cs eficaz, en el tratamiento de mantención, de las leucemias linfoblásticas. Se usa también on cl trat:micu. to del Osteosarcoma pero en dosis altas. Por vía intratecal está indicado en tratamiento profiláctico o curativo del compromiso del SNC en leucemias linfoblásticas y linfom:s no Holgking.

Los efectos tóxicos principales son: Ulceras bucales, mielosupresión, hepatitis, ostcoporosis, Jeucoencefalopatia cuando se adninistra por vfa in:ratecal y neumonitis (alergia).

Su acción citotóxica es contrarrestada jor cl factor citrovarum o áciclo folinico y este eferto se usit en clínicat, cuando se necesita idministrar M.T.X. intratecal aún en presencia de efectos toxicos generales o on el Osteositcom en que se indican closis muy att: 100 y $200 \mathrm{mg} / \mathrm{kgr}$ y luego se inyecta ácikto folinico \$-6 horas después impidiendo así los ciectos tóxicos sobre el hoéspert.

6 Mrapropurino. Es un antipurínico descubierto en 1951. Se usit en tratamiento de mantención de letucemia linfollásticn. Es poco tóxico y se puedle administrar durante um largo periodo. Es capaz de producir leucope. nia.

5 Fluor-tracilo. Es análogo de las pirimílinas.

Sc usa en algunos carcinomas.

I as manifestacioncs de toxicielal son fre. ruentes: náuscas, vómitos, diarrea, cstontatitis, citopenia sanguíneal,

Citosima arabinósido. También es análogo de las pirimidinas. Se indica especialmente cn las leucemias linfo $y$ mieloblásticas y en algunos linfornas.

Un efccto importante es la inhibición de la progresión cle Jas células a través del ciclo celular y este hecho es muy importante en al diseño de combinaciones quimioterápicas. lit mecanismo responsible de este electo no se conoce tyicn.

Los principales efectos tóxicos son: megaloblastosis, aplasia medular, trastomos tliges. tivos. Debe administrarse cuidadosamente en presencia de daño hepático porque esta droga se excreta primariamente por el hígado. 


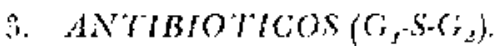

Son drogas fase especificas y fundamontaimente actuan en la síntesis c!el Aon.

I os principates son:

Arimonicina b. Es protucicla por varias espe. ciss ele Streptomyes ; si descubrió en Igto pero sus efectos citotóxicos be encontraron en 1052. Se usat especiamente en Tumor de Wil. ms, Rabcomiasiroma y Saroma de Ewing.

Entre los principales sintomats tóxicox cstán: uccraciones y nerosis de la boca, wastornos dige tivos, aplasia melular, aloperia. pigmenlación cutinea. Fsta drogit amenta lo' efectos letales de las irtadiaciones y cotas deben redtlcirso para prevenir intensa toxicidal solue los iejidus irratiados. Se elimina principalmente por el higatos por lo que laty que usirlit adialosamente si hay daño hepatio patevio.

Dannorubidomicint. Se clecubrió simulátneamer'e en Jtolia y Franciat en 1562. Es producida por el streptonyces pencetius y coruleo-rtibidos. Se usi principalmente en la indurcion de la remisión an letucemists, egualis, linfo y miejoblásticis.

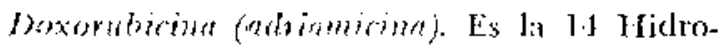
xictanorubitomicina $y$ se aisló en 1967 elel mimno Streptomyces pencetius variedad cacsins por al tabotatorio Filmiatia de Milín.

F,n perliarial sus indiaciones son máts anplias que el malicamento anterior y se está tesando an tratamiento de leucemias y varios tumotes só]idos (Nefroblasioma-Neumblastomas. Ralktomiosatconas, Osteosarema, etc.)

F.stos dos últinos antibiótices, azlemits do los defector tóxicos generales: mielosupresion. alopecia. tiastormos gaktrointesinales, producen cortiomiopatía nanto se ahministran durante un liempo largo y por es'o la dosis míxima total de la Adriamicina no debe ser superior a 550-600 mg/ $\mathrm{m}^{2}$. Esta se cxoreta principalmonte por el higado y puede dar una coloración rojiza en la orina que no tiene. importancial.

\section{ALCALOHOLS DE PLANTAS.}

Vincristina (Oncovin) y Vinblastina (M), deriviclas de lit planta periwinkla. vinca rosea. fienen una amplia aplicación en cancerología inlantil: leuremias agulas, linfomas, tuno res embrionarios, cte. Actúan en la mitosis del ciclo celular (drogrs fase específicas) inhibiendo el perítje dé cólulas en la merafase. Produ. cen neutoloxicidad con dolor intenso cn extre. mixlates inferiores, especialmente la Vincristina, pero lo interesante es que este problema disninuye on calla closis subsiguiente y generalmente desajpanere después de la ya o 4 a do$\therefore$ is. Otoss problemas som: constipacion que si es muy intensa doben disminuirst las dosis siguientes, depresión mental, convulsiones $y$ aún coma, que a veces pueden ser mortales. concrabmente no producen depresion mexlulaj pero si alopecia, adcmás Liponatremia con orini tripercinica y retención cle agua, debisto id intelectada secreción de homona antiduréticat.

sc clininau por el hígado y dehen usarse arutelosimente en presencia de daño hepático.

\section{ENZIMAS.}

I.Asparaginasa lis la prinera enzima cmpledfla con éxiro en el tratamiento de una enfermedarl maligna. Lal aspatagint es un aninó́riclo necesario para la vícla de las células. Las colutas nomales tienen dos maneras de oftenerl., o bien Jis loman de la sangre o bien las sintetizan. Las celnlas leucimicas son incipaces de sintetizarla. Is L'asparaginasa produce una deficiencia aguda de asparagina en el plasmá por metabolización de ella en áciclo aspútico y atmonio. Las rélulas leuré micas privadas de su fucnte de asparagina, mucren. Eslos heches son muy importantes ya que. por primera ves, el tratumiento cle una Jeucemia está basado en la existencia do uma dilerente actividad bioquinica entre célula leiccimica y normal.

La I'Asparaginas actu en lase $f$, del $\mathbf{c}$ clo celukar y se uha en el tratamiento de inducción (te leucenta linfoblastion, asociach ron VOK y Prednisona. 
Produce hipersensibilidad debido a impurezas en la preparación de eśa enzima y la reacción puede fluctuar desde critema transitorio hasta muerte. Puctle adcmás, producir hipoalbuminemia y toxicjdad pancreatica.

\section{ESTEROIDES.}

Se usa especialmente la Prednisona. Produce lisis te los linfoblastos y linfocitos e inliben la proliferación del tejiclo linfítico. Pueden atravesar Ja barrera hematoencefálica. Sins indicaciones principales son: tratamiento de inducción de la lencernia linfoblástica y en linfomits.

Sus efectos colaterales se cleben a lis dosis farmacológicas que se usan: Síndione de Cushing. hipernatremia, hipokalenia, osteoporosis, hipertensión. De todas las drogas que se utilizan en el tratamiento de los cánceres, los esteroides son los que protucen mais inumosupresión.

\section{MISCEIANEOS.}

Ssilo nos relerimos a la Procatbarina. Derivaclo dc la Metilliclrazina, se sintetixó en 1962. Su mecanismo de acción no se conoce bien pero se ha demostrialo que inhibe las sintesis dc proteinas, ARV y ADN. Se indica en linfomas. Los efectos tóxicos son: miclosupresión y expecialmente trombopenia, nanseas. vómi(35. depresión o hiperexitibilictad, dematitis.

ASOCIACION DE DROGAS (Poliquimiote. rapitt.

La quimioterapia múltiple, simúltúnca os sccrencial, es la que ha producislo el mejor resultado actuil en el tratamiento de las cofer. medlades malignas: atmento de sobrevidia $y$ probable curación de leucemia linfoblástica, de enfermedad de Hodgkin, tumores embrionarios etc.

Se ha remostrado que lis drogas ukadas individualmente pueden obtener 5 a $15 \%$ de remisiones do los tumores sólidks, según el tipo de tumor. estado cvolutivo de la enfermeclad, criterios ele remisión escogidos y $50 \%$ cn leacemias linfoblásticas, mientras que la con- binación de ellas permite obtener no sólo remisión en un porcentaje mayor: 80 a $90 \%$, sino inclesso curarlos.

Empíricamente la estrategia de estas comlinaciones de drogas es aumentar el efecto intitumoral sin aumentar los efectos tóxicos.

Los principios que clirigen lat elaboración de combinaciones de drogas que se potencialìan son los siguientes:

1. Asociar drogas que usadas solas han demostrado ser eficaces sobre los tumores.

2. Asociar clrogas que se potencializan en lis secuencia escogida.

3. Utilizar drogas ayo mecanismo de acción sea diferente.

4. Usar drogas cuya toxiciclad predominante ocurra en diferentes tejiclos y en distintos tiem. jou despucis de sil administración.

5. Uilizar un prolocolo de tratamiento intermitente y prolongado ya que así se obtienen menos efectos mielo $\epsilon$ inmunosupresivos, que las quimioterapias continuas.

\section{RESULTADOS}

lil Centro de Oncologia del Hospital Luis Calvo Mackenna inició sus actividades en cnero de 1968. Descle entonces y tast: agosto de 1973 ha atendido 242 enfermos menores de 14 años con leucemia y tumores sólidos malignos. En las Tablas 4 y 5 se analizan los resiltades dos años dexpués (agosto 1975) y se incluyen todos los enfermos de cualquier grado clinico, incluso aquellos que fallecieron antes de iniciar el tratamiento.

En esta estadística se excluyen los tumores (lel S.N.C. yat que se atienden en centros de neurocirugia. Los retinoblastomas corresponden a grados clínicos avanzalos que requieren tratamiento quimioterápico.

Entre los 242 enfermos hay 67 leucemias $(27,69 \%)$ y 175 tumores solidos malignos $(72,31 \%)$

De 50 enfermos con leucemia linfoll]ástica 11 sobreviven más de 2 años $(22 \%)$ y dos de éstos más de 5 años quienes están sin tratamiento mís de 2 años. En cambio, de 17 pacientes con leucemia mieloblástica, ninguno 
PACIENTES INGRESADOS ENTRE ENERO $1969 \mathrm{~V}$ AGOSTO 1979

(ANALIZADOS EN ACOSTO ]975)

CFNTRO ONCOLOGIA, HOSPITAI LUS CALVO MACKENNA

\begin{tabular}{|c|c|c|c|}
\hline & ritios & Fallecidos & Total \\
\hline Icucculia Linfolblástica & 11 & $\$ 9$ & 50 \\
\hline Ie:ucernia Micholslistitid & 0 & 17 & 17 \\
\hline I.inforatcomat & B & 13 & 21 \\
\hline Burkitr & 4 & 7 & 11 \\
\hline Reticulosarcottra & 2 & 6 & 8 \\
\hline Irodgkin & 17 & 17 & 34 \\
\hline Nefroblastona & 9 & 9 & In \\
\hline Neuroblastomal & 4 & 8 & 12 \\
\hline Histiocilosis $\mathrm{X}$ & 15 & 4 & 19 \\
\hline Tiratoma Maligno & 3 & $\therefore$ & \& \\
\hline Gatrcinoma & 4 & 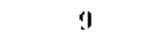 & 13 \\
\hline Sarcoma de partes Julandas & 3 & 13 & 16 \\
\hline Retirsoblasisumat & 3 & 5 & 8 \\
\hline Osteosirconia & 1 & $\mathbf{I}$ & 2 \\
\hline Sutcolud de Ewing & 1 & J & 2 \\
\hline Hisceltínea & 2 & 1 & $\$$ \\
\hline
\end{tabular}

Iabla $\times 15$

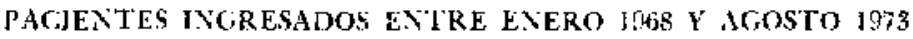

(ANALIZADOS EN ACIOSTO 1975)

CENTRO ONCOIOGRA. HOSPIJUL IIIS CALSO MACKENNA

\begin{tabular}{|c|c|c|c|c|c|c|}
\hline & \multicolumn{2}{|c|}{ Viruos } & \multicolumn{2}{|c|}{ Fallecidos } & \multicolumn{2}{|c|}{ Total } \\
\hline & $N$ & $\because$ & $x$ & $\pi$ & $N$ & $\%$ \\
\hline Iencruias & 1] & 16.4 & $5 \sqrt{3}$ & 83.6 & 67 & 100 \\
\hline lumores sólidos & 76 & 43,4 & 99 & $\breve{j 6,6}$ & 17,5 & 100 \\
\hline Total & 87 & 35.9 & 15.5 & 641 & 242 & 100 \\
\hline
\end{tabular}

sobrevive, lo que consuerda con el pésimo pronóstico de esta enfermedacl.

De los 175 niños con tumores sólidos, 76 sobreviven más de 2 años $(43,4 \%)$ annque algunos aún padecen de enfermedades activas (Enf, de Hodgkin, carcinomas).

En la tabla 4 vemos que el $50 \%$ de los enfumos con tumur de Wilms, solmoviven 2 años, sin evidencia de enfermedad y se consideran cumalos. El resultado de los otros tumores malignos se puecle ver en la misma tabla.

Para mejorar estos resultalos es neces:trio insititir en el diagnústico más oportuno de estas enfermedades, $\in$ n el cual juega un papel fun. damental el pediatra general, puesio que rea. liza controles de salud sistemáticos a lactan. ies y minos mayores. 
1. Fxelby, Pft. R, Other alutominal tumors. Cancer 35: 910. 1975.

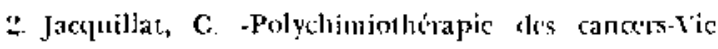
Nedicale, 4, 1974 .

3. Kump, C., Schnaufer $\mathrm{l}$. The managenent of abelominal neuroblatstoma, Cancer 35: 905, 1955.

4. Mathe, G., Buricl. J. L... Scliwargemberg. I. Schneikler. M., Catuan, A., Schlumbergert, J. R., Hayat, M. ec De Wassal, $\mathrm{F}$. Active inmmo therapy for acme: lituphoid ketikemia-lancet i: 607, 1969.

5. Mathe, (;. Currents status of inmunothcraps of hu- man cancers: lcukemia. lymphomas, solid tumorsDruggs 8: 411. 1974.

1. Murphy, M. L. The multidiscipline team in a catsaer center-Caner 35: 876, 1975.

7. Vietıi, T. y Edelstein M. Pediatric chcmolherapyperi. Annals 4: 76, 1975 .

8. Wolff. J. A. Advances in the treatunent of W'ilms tumor-Ciancer 35: 001, 1975.

p. Ziegler, J. L. Morrow, R. H. Fiss, I., Kyalwazi, S. K. y Carbone, P. P. Ireatment of l3urkitt's tumor with Cyclophosphanide Cancer 26: 474, 1950 . 